\title{
LA PEDAGOGÍA EN EL UMBRAL DE LA CIENCIA
}

\author{
Marco Emilio Bautista Buitrago \\ Magister en Educación Universidad Santo Tomás, Bucaramanga \\ marcoebautista@gmail.co
}

Resumen

El artículo desarrolla esencialmente cuatro aspectos: considerar que desde la modernidad, la razón y la práctica en alianza se constituyeron en un problema de manejo para la educación. Segundo, manifestar los efectos descomunales que la razón moderna con apoyo de la educación promueve a espaldas de la pedagogía. Tercero, despertar el interés por el valor de la pedagogía en la enseñanza de cualquier disciplina en aras a hallar la ciencia y por último reconocer que la pedagogía fue y ha de ser fundamento y senda de la ciencia, razón por la que asciende como ciencia, siendo esta idea a su vez, eje de análisis y reexión a lo largo del texto.

Palabras Clave: Educación, pedagogía, razón cientícotécnica, producción, consumo, práctica reexiva, ciencia.

\section{Summary}

This article will examine the role of pedagogy in the threshold of science from four dierent aspects: rst, to consider reason and praxis in an alliance, constitute themselves as a problem of management for education. Second, to check out the huge impact that modern reason, with the education support, promotes to the pedagogy. Third, to become interested in the essential value of pedagogy when teaching any discipline, in order to nd science and nally to recognize that pedagogy was and should be the foundation and path of science, this is why pedagogy stands as a science, the crux of the analysis and reection throughout the whole text.

Key words: Education, pedagogy, technical-scientic reason, production, consumption, reective practice, science

\section{INTRODUCCIÓN}

El presente artículo busca exponer algunas circunstancias que la modernidad ha generado sobre la educación y la pedagogía; estas circunstancias emergen de un acto de reexión a partir de experiencias de vida, de lectura de textos y de análisis cotidianos del acontecer educativo, social, cultural, económico y político que acaecen en Colombia y el mundo, donde la educación ha tenido un papel preponderante de responsabilidad y culpabilidad sobre los hechos desarrollados a lo largo de los dos últimos periodos(modernidad y posmodernidad) de la historia humana.

A raíz de este acto reexivo, se logran desarrollar cuatro aspectos:

Alianza entre razón y práctica un problema de manejo para la educación. Este aspecto a pesar de ser un hecho abordado en distintos momentos por distintos académicos o estudiosos, no deja de ser actual, razón por la que el texto deja en claro que una vez la razón toma las riendas de la historia, ésta ve que no puede

en su condición libre, busca alianza en una práctica basada en una lógica matemática sin medir consecuencias, de las que la humanidad hasta hoy no se ha logrado limpiar, dejando a su paso un vacío ético.

Efectos de la razón moderna y de la educación a espaldas de la pedagogía. Por una parte invita a recuperar la misión de la educación y hacer de esta una posibilidad para todos, desde la que se cultive más que el amor por el lucro y el consumo, el amor por la sabiduría, el conocimiento, el sentido y valor de lo humano. Por otra parte denota cómo la educación se ha constituido en una práctica demoledora de la cultura, induciendo a que pocos crean en ella a raíz de que conlleva a actuar por fuera de los parámetros humanos, constituyéndose en aliada de sistemas adúlteros. 
3. La pedagogía promueve identidad y da sentido. La educación desde la pedagogía constituye un peligro para el sistema vigente al desarrollarse coherentemente, por ello, absurdamente, a raíz de esta situación la pedagogía entra al anonimato, desarrollándose cualquier cantidad de "planes o programas educativos" sin contar con la aprobación de ésta, de ahí, el número de profesionales de mantenimiento que gradualmente reciben título sin ofrecer resultados, tan solo a la espera del lucro, el cual no es educable. A raíz de esta situación el texto pone de maniesto la importancia que ha tenido la práctica pedagógica en cuanto que avecina al estudiante (futuro profesional) al umbral de la ciencia y con ella la investigación.

4. La pedagogía, fundamento imprescindible de la ciencia. Se reconoce la condición subjetiva de la pedagogía, a su vez que, no se desaprueba su trabajo objetivo en cuanto que para la ciencia cualquier dato de referencia o emergente dentro del proceso es objeto de explicación, motivo por el cual todo resultado no escapa a la interpretación, acto que obliga al buen uso de la reexión. En consecuencia hacer buen uso de la reexión es hacer pedagogía y, hacer pedagogía es hallar y promover conocimiento en una dinámica de espiral, donde la promoción del ser humano ha de ser causa y efecto al mismo tiempo, pues solo quien trabaje con pedagogía comprenderá su misión.

\section{DESARROLLO}

1. Alianza entre razón y práctica un problema de manejo para la educación

Aliarse la razón a la práctica sobre un mismo interés, fue hallar la llave de la caja de Pandora para ocasionar una crisis ontológica y epistemológica a partir del desarrollo de esquemas lógicos, matemáticos, sobre sistemas comple- jos. Abrir la caja, fue lesionar las ciencias del espíritu, (ciencias sociales y humanas), una herida que progresivamente lacera y debilita su esencia hasta hacer de estas un instrumento de interés racional y productivo. Es un maltrato que promueve la razón moderna y que golpea a la pedagogía, y en efecto a la educación; de ahí la pregunta: ¿Para qué la educación si lo que se necesita es trabajar y obtener dinero? De modo que la pedagogía constituida como"el pensar de nuestras acciones educativas y el preguntarnos acerca del sentido de lo que hacemos al educar" (Unigarro,1999:19), sacrica su misión y deja de ser senda por la que transitan las ciencias, a pesar de verse asediada por el espíritu de las ciencias naturales, trayendo con ello una transformación humana ceñida de crisis ética y sociocultural.

Con la explosión de la razón moderna la educación ha caído presa y confundida en ella misma. En consecuencia, recibe como castigo, abandonar su misión. "La razón, además, hizo que las personas ocultaran sus sentimientos $y$ que, incluso el arte perdiera la posibilidad de ser una expresión no sometida al rigor de la lógica" (Unigarro, 1999:114) Por lo mismo se comprende que la educación se aparte de su tarea, la de acompañar el proceso de formación integral de vida del ser humano; ya lo decía Fischer (1971) "La educación es un hecho de la vida histórica y social de los hombres" (citado por Unigarro, 1999: 38).

Por supuesto que la educación es un instrumento o mediación de la razón cientíco-técnica, por ello altera su misión, pasa de hacer con el ser humano una labor integral a hacer con la razón cientíca una acción de desarraigo social y humano. A partir de ello mujeres y hombres se entienden no en cuanto son, sino en cuanto hacen. "La educación es una práctica social y se da en el interior de la sociedad" (Unigarro, 1999:41-42); ésta favorece los procesos de desintegración humana mediante el uso adecuado de la razón cientíca en aras al desarrollo material, en los que producción y consumo excesivo, 


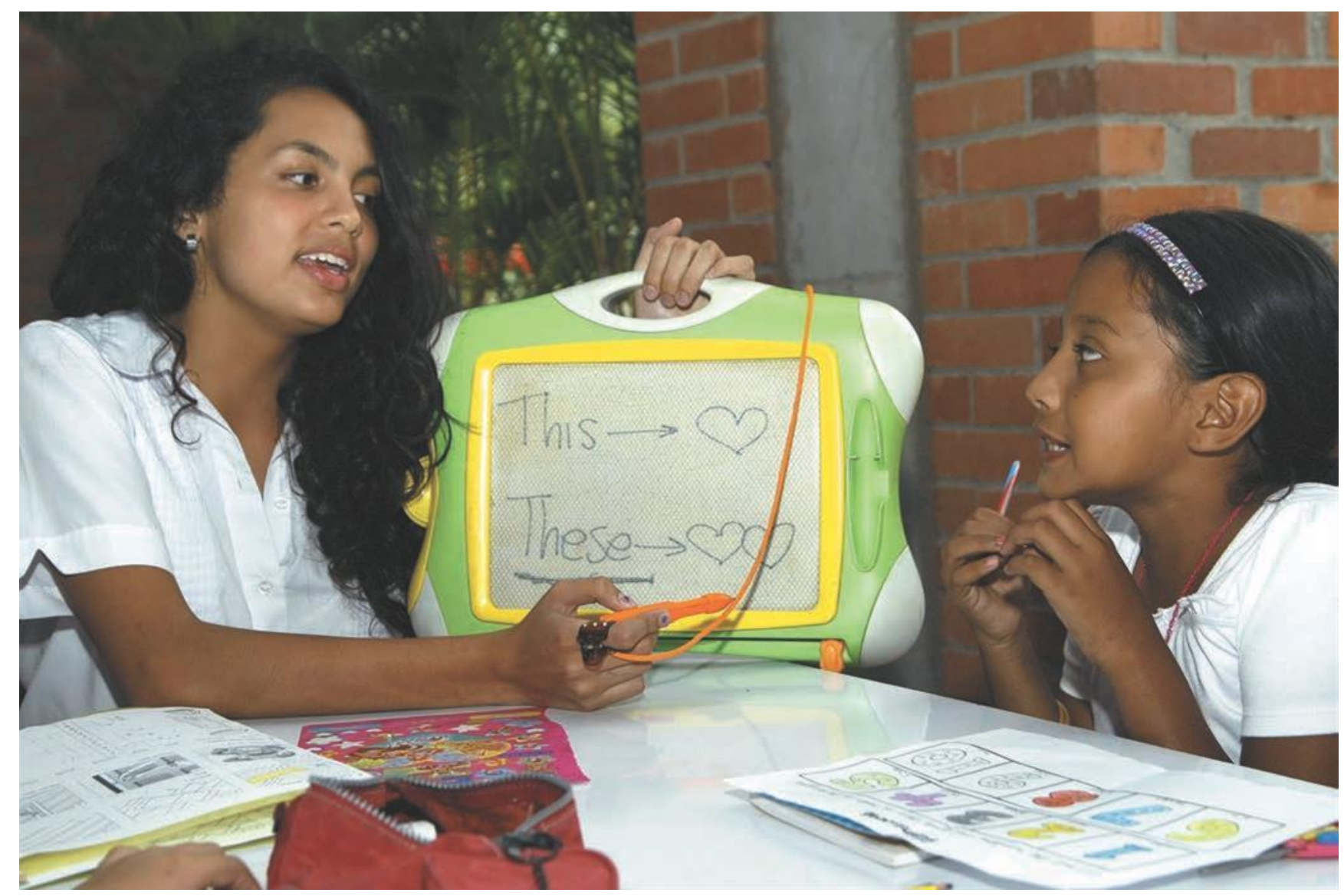

son fuente de vida o muerte del ser humano. Por consiguiente quien está en condiciones de producir, vive, pero... ¿Quien no produce? Esta ha sido desde entonces la nalidad de la razón moderna y con ella la educación. ¿Qué hacer ante ello? ¿Involucrarnos o resistir?

Así, quien está en condiciones de hacer parte de este estilo de vida, de esta cultura, se interesa mucho más por formarse dentro del marco de la ciencia y la técnica, la producción y el consumo, aspirando a un deseo de poder, fortuna y prestigio. Preocupa desde entonces que se pretenda enfocar la esencia de la educación en desarrollar habilidades técnicas, que aunque necesarias no han de ser la prioridad.

Hacer parte del estilo de vida de la cultura cientíco-técnica, es entrar en la necesidad de evitar y tomar distancia del marco esencial de la educación; de tal forma que, formarse para la vida, fortalecer y desarrollar valores, moderar los sentimientos, velar por el patrimonio sociocultural, fortalecer y promover identidad regional y nacional, proteger los recursos naturales, fortalecer los lazos familiares, sociales y de convivencia entre otros. no están dentro del esquema de prioridad de las ciencias técnicas. Esto en virtud de que

La educación se entiende como toda clase de hechos 0 acontecimientos que se dan alrededor de las personas y que producen cambios en ellas (...) abarca no solo las inuencias interhumanas sino también el conjunto de las inuencias no personales del entorno material, del paisaje, del clima, del espacio" (Schneider,1953 citado por Unigarro, 1999:38).

Desde este horizonte cientíco-técnico, la población colombiana presenta vacíos en su educación, acaso: ¿Quién educa hoy en Colombia? ¿De dónde salen las políticas educativas, y éstas a su vez a qué políticas internacionales responden? Se trata de una cadena de poderes 
que estructura estas políticas internacionales desplegadas en los escenarios de la familia, favoreciendo la política del no dar tiempo para pensar, sino la promoción de actividades que ocupan a la población en la producción mal llamada utilitaria, por temor a que pensar libera.

Cuando tenemos dos seres humanos juntos tenemos la posibilidad de que se enfrenten y se neutralicen, tenemos la posibilidad de que se alíen, tenemos la posibilidad de cada uno de ellos transforme al otro, tenemos incluso la posibilidad de que se multipliquen. Para este $n$ no nos sirven las simples verdades de la aritmética ni las comunes verdades de la estadística (Ospina, 2012: 21)

A raíz de lo manifestado por Ospina, lo individual y colectivo, se enfoca en el mercado, $<$ hacer >, por que <no hay tiempo para pensar>, además que se promueve esa actitud como expresión cultural. De tal forma que quien estudia, lo hace en función de una escala de poder, producción y consumo. De ahí que "(...) las cosas ya no existen para ser sabidas sino para ser consumidas(...) ya se sabe que después de usar el vaso hay que destruirlo enseguida" (Ospina, 2012: 16). Frente a esta máquina demoledora de humanos y de culturas, no sobra recordar que "El dominio material de la pedagogía, (...) no son los animales, ni las plantas ni los números, es exclusivamente la persona" (Unigarro, 1999:15)

De modo que urge defender y promover hoy una educación que piense al ser en los otros. Educar hoy, implica y compromete la vida, el no educar es castigar al hombre y dar muerte a la vida. Porque después de esto, qué esperar ¿Una reforma? De tal modo que hoy la "educación" ha caído en un adiestramiento, que nalmente busca enseñar mecanismos, fórmulas, estrategias que permiten sobrevivir. De ahí la pregunta cuando alguien aspira a un trabajo: ¿Usted qué sabe hacer?

Por la apremiante necesidad de la formación cientíco técnica, la escuela corre el peligro de limitar las capacidades intelectuales a las funciones ocupacionales, olvidando que son los hombres y mujeres en relación social, los que construyen la ciencia y la tecnología(...) (Unigarro, 1999: 119)

He ahí la crisis de la educación y con ella la pedagogía, porque al parecer los mecanismos para aprender ya están dados, tan sólo basta seguir el catálogo, la receta y las metas: ¿Para qué didáctica o para qué pedagogía, más cuando éstas me conminan a reexionar sobre mi quehacer? Hoy: ¿Para qué reexionar, mucho menos para qué pensar si eso no da para mantener ahíta la nevera en el hogar? Recordemos que la educación actual imposibilita el pensar, promueve la cultura del analfabetismo profesional, hoy lo mejor es hacer, no ser. De tal forma que el docente pone a hacer a sus estudiantes, poco los confronta, poco reexiona su práctica, de ahí que la investigación misma aún no alcance el nivel deseado.

Los resultados de este planteamiento en curso son evidentes, basta reconocer lo que acontece cruelmente en este país y ver la "reacción ecaz e inmediata del marco institucional y profesional", su ecacia es vergonzosa, así lo sienten los mismos colombianos. ¡Qué decir del marco institucional internacional! Es una actitud ignominiosa, que sorprende y enceguece con actos deshumanizantes. Frente a esto: ¿Cuál debería ser la nueva dinámica de la educación nacional para recuperar su ser esencial y resistir a la fuerza de la razón cientíco-técnica que opera bajo el manto de las políticas educativas internacionales en los que al parecer mercado y consumo son el horizonte de sentido y de vida, pasando por alto lo sociocultural y lo humano?

2. Efectos de la razón moderna y de la educación a espaldas de la pedagogía

Emerge la necesidad de recuperar la misión de la educación desde cada uno de los carismas para lo que cada institución fue fundada. De 
ahí el interés de profundizar, dar operatividad y vida al proyecto educativo institucional (PEI), como espíritu que dinamiza y alimenta la vida integral de quienes deciden educar en una misión a quienes igualmente se identican con ese espíritu. La institución educativa y el Estado en primer lugar, no deben erradicar de su práctica el sentido humano que proporcionan las ciencias,

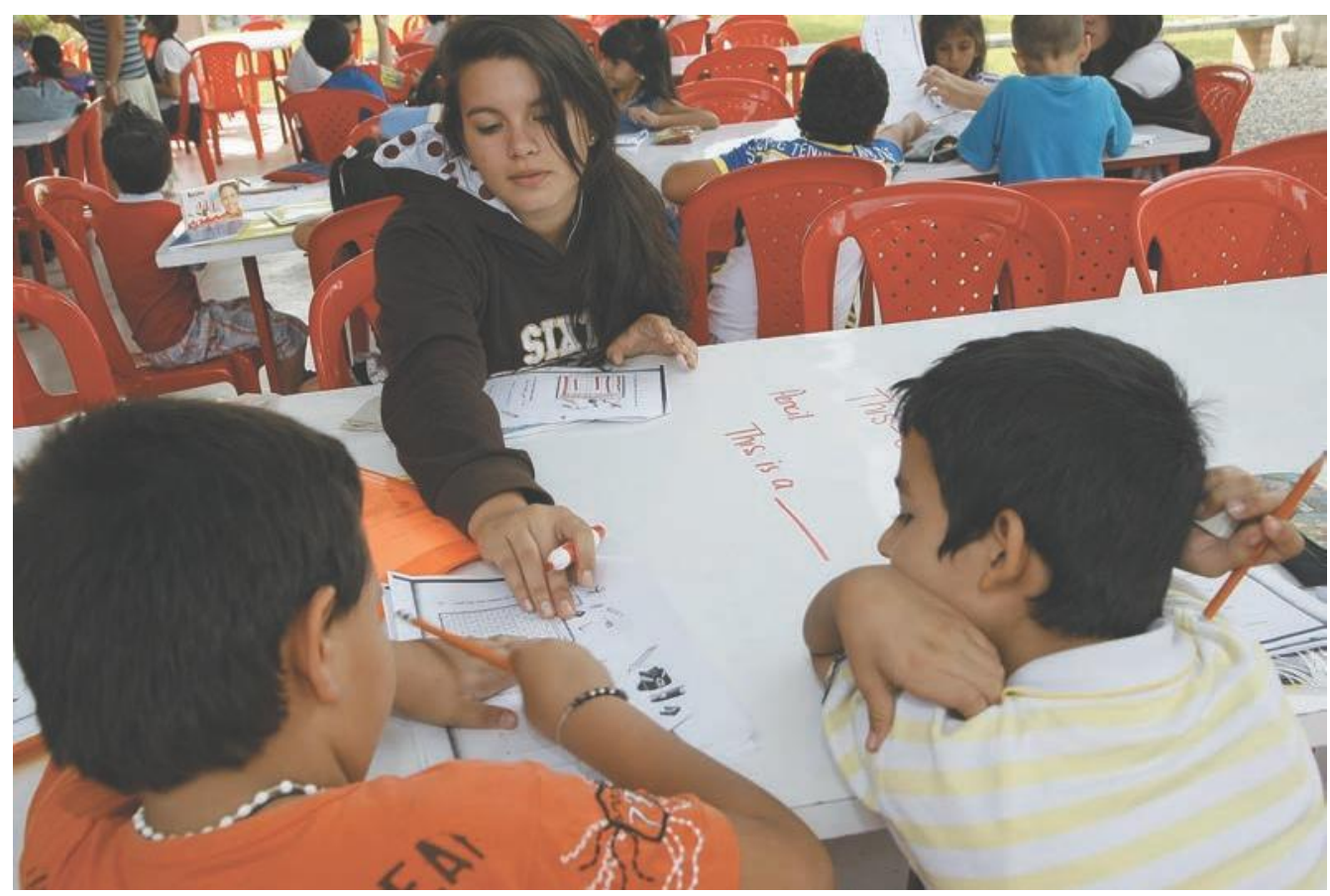
cualquiera que esta sea. Cada ciencia es un homenaje de grandeza y vida del ser humano.

La educación es un concepto que designa algo más que crianza, adiestramiento o instrucción. Es una acción que se realiza entre personas (no ya entre objetos y personas, como en el caso de la instrucción) y que consiste en la inuencia de unos sujetos sobre otros con la nalidad de brindar espacios de formación(...)" (Unigarro, 1999: 41-42).

Esto mismo ha de transmitirse desde que se enseña y se aprende, cada ciencia es y ha de ser un oasis de tranquilidad para la familia y la población humana, una fuente a la que todos deberíamos acudir sin miedo, pero lamentablemente se ha vuelto una realidad desesperante y lejana a la que pocos alcanzan; primero, por razones económicas y segundo, por desconocimiento de la misma disciplina.

Actualmente la escasez de dinero conlleva a que pocos usen los servicios para los que cada disciplina ha nacido y aunque pudiendo aprovecharlos, se teme que no respondan por desconocimiento de la misma; de ahí la crisis de la ética profesional. Desde los sesentas y setentas

el fenómeno contracultural "critica severamente el discurso normativo que se pronuncia en la escuela y la estructuración de una vida cticia que allí se genera" (Unigarro,1999: 112) Problemas en sí que no son resueltos porque ha predominado más el amor por el lucro que por el conocimiento, por lo mismo acciones resueltas más por vía de la trampa o del atajo.

Basta dar una mirada a los últimos resultados de las Pruebas Saber en los niveles de Educación Superior para evidenciar entre líneas lo que es, hay y se hace en Colombia (www.icfes. gov.co. Principales resultados en los módulos de competencias genéricas Martes, 17 de Abril de 2012), niveles muy bajos en el $98 \%$ de las profesiones a nivel de lectura y de escritura, entre otras competencias requeridas; escasamente saborean la media que es 10, se observa entonces que calamitosamente la educación está rezagada, por ello no es extraño que aore la indisciplina social. ¿Qué esperar? A raíz de lo anterior: ¿Qué pensar de la investigación en Colombia, cuando lectura y escritura fallan?

"(...) Y como quien no lee, tampoco es capaz de escribir, la producción de trabajos académicos valiosos en nuestras universidades resulta paupérrima. En la más reciente tabla de 
evaluación de institutos iberoamericanos de educación superior (Scimago), es penoso el aporte colombiano en materia de publicaciones: la entidad mejor calicada es (...)" http:// www.eltiempo.com/opinion/editoriales/articulo-web-new_nota_interior-12135934.html (7:38 p.m./16 de agosto del 2012)

Importante volver al espíritu de la educación, a su misión, a ser educados para contribuir a identicar carismas o vocaciones y desde ahí poder favorecer la cualicación de quienes se enamoran por el conocimiento y el desarrollo riguroso de una ciencia:

La disciplina de la escuela alejaría a los alumnos de los vicios, frutos de la ignorancia. Los maestros eran los cirujanos del bien que operaban las conciencias. La escuela se convirtió en la fuente donde se beben los rudimentos de las ciencias, con unos resultados salvadores para la humanidad (Unigarro, 1999:107).

Ahora bien, las ciencias se consideran equiparables en cuanto que buscan preservar al ser humano, cultivarlo en todos sus aspectos (cultural, social, político, económico, religioso(...)). Sin embargo, hay que decir que la mayoría de Estados gubernamentales, apoyados en la política de los recursos, la producción y el consumo, han malversado la misión de la educación.

¿Cómo superar una época en que la educación corre el riesgo de ser un negocio, donde la excelencia de la educación está concebida para perpetuar la desigualdad, donde la formación tiene un n puramente laboral y además no lo cumple(...)? (Ospina,2012: 31)

Es de mencionar que las políticas gubernamentales han promovido y a la vez han conducido a que la educación se constituya en una práctica demoledora e inhumana de la cultura.

"Nuestra época, convierte a los obreros en apéndices de los grandes mecanismos, en seres cuya individualidad no cuenta a la hora de ejer- citar sus destrezas, es especialmente cruel con millones de seres humanos" ( Ospina, 2012: 27).

Por consiguiente cada sujeto se ve obligado a pensar y actuar por fuera de su vocación profesional, constituyéndose en aliado de un sistema que adultera los principios de la Constitución, proponiéndose como objetivo obtener un botín que rompe con el principio de la justicia, conllevando a que las metas se logren con deslealtad. Ya lo decía Bourdieu

(...) la medida tecnocrática del rendimiento escolar supone el modelo empobrecido de un sistema que, no conociendo otros nes que los que le adjudica el sistema económico, respondería óptimamente, en cantidad y en calidad, y al menor costo, a la demanda técnica de la educación, es decir, a las necesidades del mercado de trabajo (1993: 232).

El botín obviamente lo poseen las multinacionales que se han aliado a muchas otras empresas nacionales, dueñas de la economía de un país, empresas a las que muchos aspiran. ¿Cómo llegar a ellas? Fácil. ¿Acaso no es prioridad optar por aquella disciplina que me produce y genera bienestar sin esfuerzo alguno, sin importar si me exige o no vocación? La pregunta encuentra respuesta en el ansia y necesidad de lucro que hoy se promueve. Acaso: ¿No es esta una forma fácil de aniquilar al otro, de pasar aún por encima de su vocación y servicio?

Así, en los países en los que la tasa de analfabetismo es muy elevada, el simple hecho de saber leer y escribir o, a fortiori, la posesión de un diploma elemental basta para asegurar una ventaja decisiva en la competencia profesional(...)"(Bourdieu, 1993: 233).

Por ello, muchos que sin tener vocación estudian lo que no creen y hacen lo que no sienten, produciendo como efecto una deshumanización generalizada.

"No se trata de escoger profesiones rentables sino de volver rentable cualquier pro- 


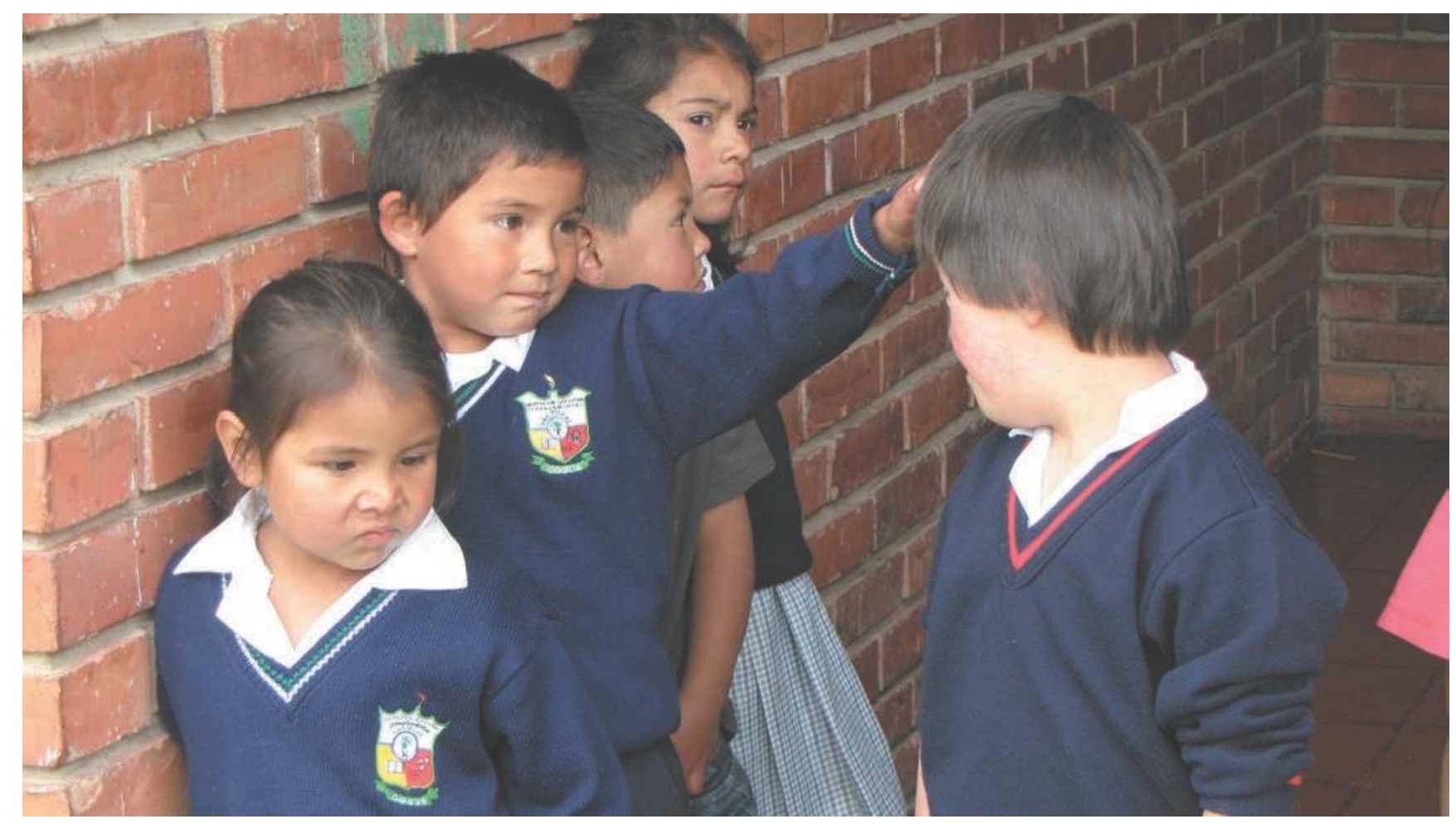

fesión, por el hecho de que se la ejerce con pasión, con placer, con imaginación y con recursividad" (Ospina,2012: 27), Se trata de recuperar la misión de la educación, de una educación que no pierda el enfoque del desarrollo esencial de la vocación profesional, articulada al servicio social y al cuidado de la vida donde el lucro no sea lo esencial, sino el cuidado por el ser con el otro. A propósito, William Ospina reriéndose a Mary Wollstonecraft, esposa del fallecido poeta Percy Bysshe, mani esta que ella, llevó a su hijo a un colegio de Inglaterra y al llegar preguntó sobre los criterios de la educación en esa institución: "Aquí enseñamos a los niños a creer en sí mismos, - a la vez que ella cuestionaba - Yo preferiría que los enseñaran a entenderse con los demás" Ospina, 2012: 19).

De todas formas el conicto entre el lucro y el cuidado por el ser con el otro, ha sido un fenómeno que desata miradas maliciosas, devoradoras y despreciables desde las ciencias positivas y miradas lánguidas y acuciantes desde las ciencias humanas y del espíritu. Razones despreciables de las ciencias prácticas hacia las humanas como, no aportan ciencia en sí mismas, no son productivas y empobrecen. William Ospina, para referirse a este aspecto, se apoya en una idea de Estanislao Zuleta quien maniesta que "la sociedad industrial se caracteriza por su mayor racionalidad en el detalle y la mayor irracionalidad en el conjunto" (Ospina, 2012: 12) Es un problema que emerge aún desde antes de la modernidad, he ahí la lesión a la educación, la cual ha vivido con una lanza colgada en tal situación amenazante que, en cuanto se mueve, ésta le hiere cada vez más. La educación pues, se ha constituido en la servidora de la ciencia, a su vez que en instrumento de poder. Es de creer que desde la modernidad, el tiempo para la educación ha sido dedicado para recongurar el espíritu y la vocación de los sujetos.

Por lo anterior, se ha creado y fortalecido la idea que, la educación no es rentable, que empobrece y quita tiempo. De tal forma que es mejor instruir que educar, por tanto, lo que se ha considerado "educación" básicamente ha sido un tiempo de adiestramiento; "La escuela puede darnos conocimientos y destrezas, pero a ello no lo llamaremos en sentido estricto 
educación sino adiestramiento" ( Ospina, 2012: 26). Forma adiestra en poco tiempo y lo prepara para hacer ocios muy puntuales, de ahí en adelante, cada ser -que no está siendo- ha de arreglárselas como pueda o le permita el conte xto cosicado en el que vive. "Mientras la educación siga siendo sólo búsqueda del saber personal o de la destreza personal, todavía no habremos encontrado el secreto de la armonía social, porque para ello no necesitamos técnicos ni operarios sino ciudadanos"(Ospina, 2012: 26)

¿Quién se responsabilizará entonces de enseñar las sanas relaciones humanas, la importancia de la convivencia, el amor por las tradiciones y la cultura, el sentido y valor del trabajo en familia y en comunidad, el sentido de pertenencia e identidad con la patria, si a estos aspectos el mismo Estado evade porque no son rentables, y a su vez considera que se expone porque la reexión humana hallará el camino de la conversión y del cambio social y humano?

3. La pedagogía promueve identidad y sentido

Lo anterior constituye el argumento para comprender lo que la pedagogía representa respecto de su valor y reconocimiento, pero a la vez la amenaza que representa para la actual época. "La pedagogía requiere de otros saberes y, a su vez, ellos necesitan de la pedagogía para construirse, de ahí que ninguna disciplina es autónoma."(Unigarro, 1999: 16). El no presentar resultados objetivos, medibles, vericables y quedarse en la reexión esencial de la práctica educativa le ha generado a la pedagogía anquilosamiento, permanecer en el anonimato y costarle socialmente el valor de su misión.

Esta situación anómala de la pedagogía, la ha tenido en riesgo, de ahí que pocos quieren saber de ella, por lo mismo no la incluyen en sus propuestas, trabajos y prácticas, de modo que la formación y la instrucción la han aislado e ignorado, desconociéndola como parte fun- damental de la educación. "Cuando el profesor no hace pedagogía, se convierte en un profesional de mantenimiento: aquel que hace cosas, pero que no sabe ni el origen, ni la nalidad de las mismas" ( Unigarro, 1999: 20).

Precisamente, un hecho que ha aquejado el proceso de educación humana es el: ¿Por qué las ciencias fácticas han tenido dicultad con la pedagogía siendo esta una actividad de reexión y práctica? ¿Habrá desconocimiento de ella? Sea lo que sea, la ciencia, cualquiera que sea, no puede prescindir de la pedagogía cuando de educación se trata. Esta misma situación traerá dicultades con el acceso a los métodos de trabajo. "

Hacer pedagogía es tener la certeza de que nuestras acciones educativas tendrán sentido. La educación con sentido es aquella que se funda en una sólida construcción de la reexión pedagógica. La educación sin la pedagogía no es más que un mero activismo sin horizonte"(Unigarro, 1999:52)

La mayoría busca formarse en una profesión y trabajar con ella, sin embargo, se evidencia una confrontación cuando desconocen la aplicación de la pedagogía en su campo, porque tal vez el interés pragmático opaca los nes sin mirar efectos sociales y humanos, sobre este mismo horizonte considero que fallaría la didáctica, los planes curriculares y la evaluación. "Las cosas demasiado gobernadas por el lucro no pueden educarnos porque están dispuestas a ofrecernos incluso cosas que atentan contra nuestra inteligencia si el negocio se salva con ellas(...)" (Ospina, 2012:17). Este acontecimiento pondría en grave riesgo la validez de los proyectos educativos institucionales (PEI), como la importancia de la Misión y Visión de cualquier institución con vocación educativa, más cuando se trata de una institución de educación superior.

De modo que la pedagogía dene la naturaleza de una institución y a la vez el éxito de cualquier programa educativo. "El saber peda- 
gógico, ese que se construye desde la pedagogía como disciplina rigurosa, es lo que nos da la identidad a quienes nos desempeñamos como maestros. Hacer pedagogía es tener la certeza de que nuestras acciones educativas tendrán sentido(...) "(Unigarro, 1999:52).

¿Cómo educar o hacer ciencia sin pedagogía? ¿No es acaso la pedagogía quien desarrolla miradas distintas en los sujetos en favor de las ciencias y de lo humano? "La creencia de que el conocimiento no es algo que se crea sino que se recibe, hace que olvidemos interrogar el mundo a partir de lo que somos, y fundar nuestras expectativas en nuestras propias necesidades."(Ospina, 2012: 27). Entonces: ¿Por qué el tácito, pero también evidente interés de nuestra época en invisibilizar la pedagogía, creyendo que sin ella los procesos de formación son mejores? De tal forma que, de aquí en adelante nos depara un gran reto, recuperar el valor esencial de la pedagogía.

Ante el reto que genera la dinámica de la modernidad mediante la consolidación de las ciencias fácticas, considero que experiencias particulares y enriquecedoras en el caso de $\mathrm{J}$. Piaget (1896-1980), de P. Freire (1921-1997), de D. Schön (1930-1997), de J. C. Melich (1961 - ) entre otros, generaron exitosos resultados, desde los cuales la pedagogía ha tomado una posición de lideraz-

go y ha defendido su función esencial en la educación.

No quiero aquí exponer tampoco lo que cada pedagogo ha desarrollado en sus áreas de trabajo, pero quiero dejar en claro que dichos pensadores han demostrado que las ciencias del espíritu y de modo particular que la pedagogía puede desarrollar métodos concretos que permiten obtener resultados vericables a escala cientíca en contextos personales, culturales, sociales e históricos, lo que motivaría sin duda enfocarnos en la misión de la educación, razón de ser de nuestro tema. Por consiguiente,

"No existe un método de investigación exclusivo de la pedagogía; principalmente ella se apoya en lo que le aportan las disciplinas que tienen que ver con lo social. Sin embargo, no es extraño encontrar estudios que se han fundamentado en sólidos métodos estadísticos o investigaciones realizadas desde el más riguroso diseño experimental". (Unigarro, 1999:17).

Es de considerar que las prácticas educativas y pedagógicas profesionales de aula o fuera de ella, posibilitan resultados concretos mediante procesos de investigación, desarrollados a la par con los mismos estudiantes; la mayor evidencia la comprobamos en los resultados obtenidos por los anteriores pedagogos e investigadores citados. Por esta razón, la práctica pedagógica logra posicionarse a nivel de ciencia. Sobre este horizonte, el análisis de Pierre Bordeau que hace considera que, el profesor no ha de ser"(...) un protector, encargado de asegurar la carrera de sus clientes, sino ante todo un maestro, encargado de asegurar la formación cientíca o

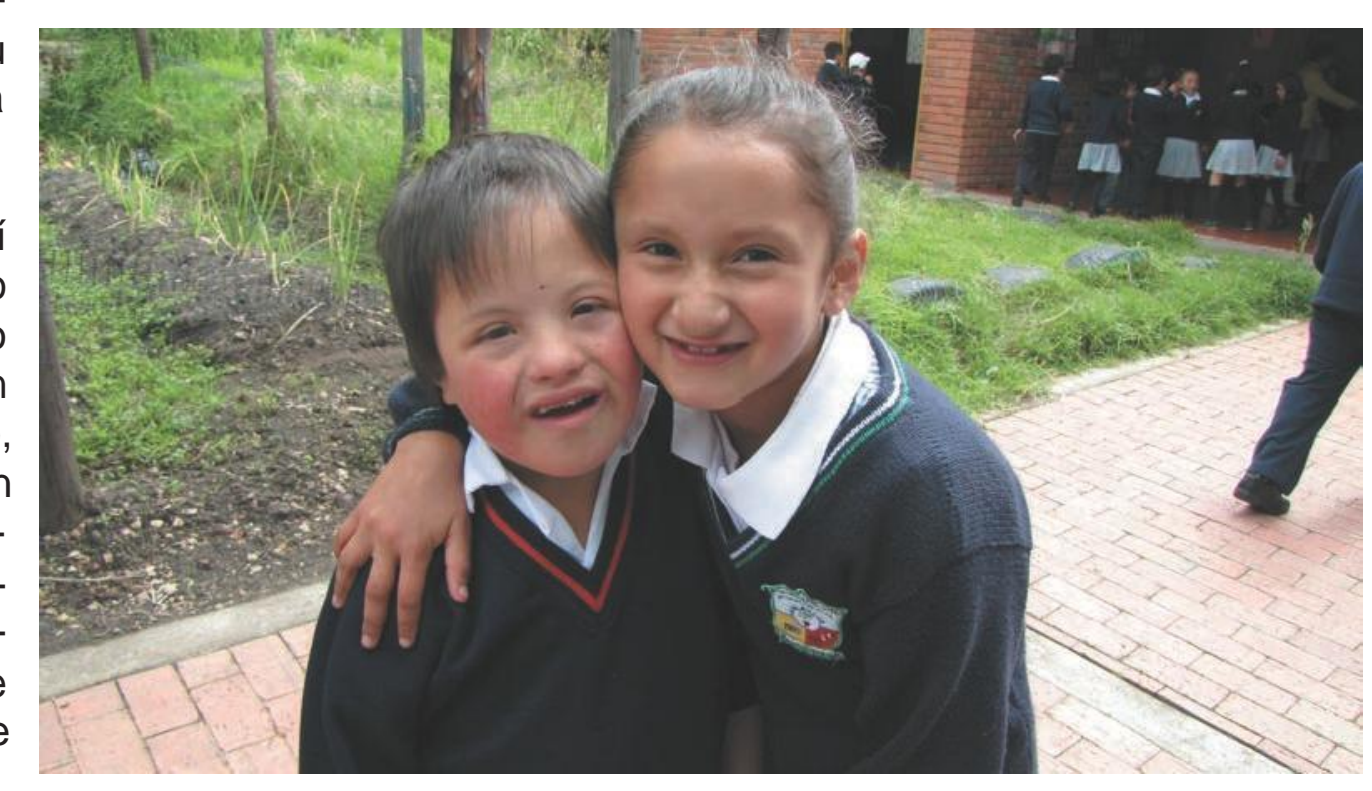


intelectual de sus alumnos o de sus discípulos(...) "(Bordieau, 2008. 80).

Para la pedagogía, aunque sus explicaciones estén articuladas por la subjetividad, no dejan de ser resultados ecaces que se constituyen a la vez en modelos o paradigmas de estudio y comprobación, desde los cuales se pueden comprender e interpretar como realidades objetivas. Los esquemas, formas de la educación, hoy han de ser objeto de revaluación.

La educación formal a veces hace pensar que las matemáticas, que la física, son un conjunto de fórmulas para ser memorizadas. Pero esas ciencias exigen mucho más que memoria, exigen que seamos capaces de razonar, de analizar, de resolver los problemas de muchas maneras distintas (Ospina, 2012: 47).

Efectivamente en esto último recae la acción pedagógica. Han sido varios los aportes cientícos desde esta perspectiva que han generado una provocación y comportamiento de grupos sociales e institucionales que, por otra parte, determinan realidades puntuales y que a su vez se constituyen en objeto de estudio de la ciencia, generando procesualmente una espiral de conocimiento. Por ello, "La educación es el gran remedio para los problemas del mundo y el aprendizaje siendo una grave responsabilidad de la especie, es a la vez, la más grande de las virtudes humanas" (Ospina, 2012: 29). Por consiguiente, será desde las prácticas mismas, desde donde la pedagogía es y será reconocida como ciencia.

4. La pedagogía, fundamento imprescindible de la ciencia

Karl Popper (1902- 1994) armaba que la ciencia no produce resultados únicos, sino que por el contrario, las hipótesis desde las que la ciencia trabaja, son posibles de falsear, lo que indica que la ciencia no sigue un único método, ni se vale de un solo análisis e interpretación. Por esta razón, se puede deducir que la pedagogía no puede seguir un único método, caería y promovería dinámicas o procesos ortodoxos con resultados que siempre la ciencia misma combatió.

Tomás Kuhn (1922- 1996), una de las guras de la investigación de mediados de los cincuenta, cuestionó las tesis de los positivistas, generando desde entonces una nueva concepción de la losofía de la ciencia centrada en factores sociológicos e históricos. Kuhn, a partir de ello introduce una nueva categoría, "paradigma". Por ello él en su obra "La Estructura de las Revoluciones Cientícas", esboza en términos generales el saber y el saber-hacer que comparte una comunidad cientíca. Si la ciencia se concibe como una rigurosa práctica humana, desarrollarla permitiría comprenderla y a su vez explicar al ser y al hombre en su ejercicio de vida. Efectivamente esa es la función de la pedagogía y en donde se halla la esencia misma de la educación. "Diez años de estudio no se pueden justicar por un cartón de grado: deberían valer por sí mismos, darnos no sólo el orgullo de ser mejores sino la felicidad de una época de nuestra vida"(Ospina, 2012: 24).

Por ejemplo, fue gran el interés de los norteamericanos por María Montessori entre los años 1911 y 1915; cabría hablar de un culto a ella. "La Montessori se consideraba a sí misma como cientíca, pero la historia de la educación no ha colocado, ni a ella ni al talentoso educador suizo Adolphe Ferriére (1879-1960), entre los cientícos" ( Holmes, 1965:170). Esta realidad, por consiguiente, es cuestionable ya que la pedagogía inicialmente estuvo sobre el camino de la ciencia, pero poco a poco la educación desde los intereses políticos económicos y mercantiles de la sociedad, fue desviando lentamente sus nes, su misión, cayendo obviamente en el servilismo de la razón cientíca. " $A$ veces me pregunto si la educación que transmite nuestro sistema educativo no es demasiado competitiva, hecha para reforzar la idea de individuo que forjó y ha fortalecido la modernidad" ( Ospina, 2012:19) ¿Qué hacer para recuperar ese apasionamiento cientíco inicial desde la acción educativa? tal vez y como propuesta, no queda otra que vol- 
ver al corazón de la educación, a su misión, con el ánimo de recuperar ese espíritu cientícopedagógico aplicado a las prácticas educativas.

Uno de los defensores de esta posición, que las prácticas sean el fundamento cientíco para la pedagogía es Donal Schön. Este pedagogocientíco considera importante discutir la práctica, identicar los puntos favorables y desfavorables, además ve imprescindible que los objetivos tengan que ver con la acción desarrollada e ir incluidos dentro de un método. Él percibe que cada profesión hace uso de cuerpos o realidades de conocimiento profesional. Lo interesante aquí, según Schön, es que cada profesión hace uso de cuerpos de conocimiento desde sus diferencias pero "también desde las similitudes que presenta" (Schön, 1998: 123), aunque "es una función de las prácticas mismas"(1998: 123), además que el profesional ha de "plantear el problema práctico como caso único"(1998: 123), esto es sin duda hacer ciencia desde la pedagogía. Solo quien trabaja con pedagogía produce; quien trabaja con pedagogía comprende su misión, comprende a sus estudiantes y estos a su vez asimilarán la fuerza y signicado de su quehacer educativo, impregnándolos de amor por la vida y por su vocación. "Mas vale enseñar procedimientos y maneras de razonar que respuestas que puedan ser copiadas"(Ospina,2012: 20).

En el marco de la práctica educativa que presenta Schön, se ubica el horizonte de la producción pedagógico cientíca. Por consiguiente, ningún estudiante se ha de comportar como si fuera a buscar claves estándares ya elaboradas sino que se empeña en descubrir características concretas de su situación problemática hasta diseñar su propuesta.

La conquista de la autonomía, el hacer uso público de la razón particular, el pensar por sí mismo y obrar en consecuencia, será el horizonte de sentido de toda acción educativa, en espacios en los que los individuos se constituyan como personas(...) (Unigarro, 1999:51).
El profesor al escuchar el problema de estudio del estudiante lo lleva a la reexión crítica, lo anima para que articule y reordene su problema, hasta acercarlo por su propio esfuerzo a hallar progresivamente nuevos sentidos de su realidad.

Consecuentes con el proceso de reexión, estudiantes y docentes escalan con disciplina sentidos, signicados, estructuras, realidades y procesos complejos desde los que probablemente despejan problemas y "construir comprensión de la situación tal y como se la encuentra(...) y sobre ella establecer un nuevo encuadre"(Schön, 1998: 124). Al respecto, Unigarro citando a Dewey (1859-1952), sostiene que

toda la educación debería ser cientíca en el sentido más riguroso de la palabra. La escuela debía convertirse en un laboratorio social donde los niños aprendieran a someter la tradición recibida a pruebas pragmáticas de la verdad; el conocimiento acumulado por la sociedad debería operar de manera palpable. Y además este debía ser un proceso continuo: la escuela debía desarrollar en el niño la competencia necesaria para resolver los problemas actuales y comprobar los planes de acción del futuro de acuerdo con un método experimental (Unigarro, 1999:96).

Para Schön el ejercicio profesional del docente, no se identica en cuanto imparta un conocimiento u ofrezca una información a los estudiantes sobre una determinada disciplina para concluir con una aplicación de pruebas psico-rígidas. Este tipo de dinámica educativa puede generar nada más que temor, insatisfacción, desencanto por la asignatura, realidad por la que la mayoría de estudiantes transita sin vibrar profesionalmente por su vocación, en cuanto que no hallan transformación. A propósito Manolo García G. citando a Mario Kaplún (1998), considera que

La educación transformadora consiste en el paso de una persona acrítica a una crítica; de 
ser pasivo, conformista, fatalista, hasta la voluntad de asumir su destino humano, desde las tendencias individuales y egoístas; hasta la apertura a los valoressolidariosycomunita rios."http://www.bantaba.ehu.es/formarse/ cheros/view/Modelos_Educativos.pdf? revision_id=80120\&package_id=80004/ 13 de julio 2012.)

Esta realidad o situación es hoy bastante preocupante, a su vez que se hace incómoda ya que el mundo de la educación más que formar humanamente en la construcción de conocimiento se ha quedado en repetir un conocimiento que supuestamente garantiza al estudiante y lo lanza al desempeño de una profesión que le va a representar un estatus social y económico como tal; considero que ese ha sido el fondo del por qué la educación se ha estancado, cayendo en un feroz formalismo y en un vacío de ser;

en nuestro tiempo lo que rige nuestras conductas no es la admiración por la virtud ni el respeto por el conocimiento(...), sino el descubrimiento ante la astucia, la fascinación ante la extravagancia, el sometimiento ante los modelos de la fama o la opulencia(...) admiramos más la fuerza que la lucidez(...)" (Ospina, 2012: 16).

Esto hace por lo mismo al estudiante un "objeto pasivo" frente a los problemas que circundan nuestras sociedades o contextos, un avariento de la riqueza, un desprevenido de los hechos y del conocimiento como un inmoral de la vida.

La educación ha de poner hoy, por tanto, una mayor atención a la labor pedagógica que desarrolla, ya sea en el aula o fuera de ella y que se hace o no explícita en las prácticas y tareas profesionales. Hoy la labor pedagógica ha de recuperar su función reexiva como generar nuevos conocimientos y actitudes válidas frente al ser social. A propósito, "en algún momento los jóvenes norteamericanos, no en- tendieron por ejemplo, cómo se los podía sacar de los claustros donde habitaba la luz de la sabiduría para llevarlos a morir en Vietnam" ( Unigarro, 1999: 112), eso es cuestionar; de modo que "Las verdades de la estadística no pueden eclipsar las verdades de la psicología o de la estética" ( Ospina, 2012: 20) como de otras disciplinas sociales.

Schön, considera que la estructura de los problemas es semejante para todas las disciplinas, además porque éstas presentan "muchas perspectivas en competencia sobre la naturaleza de la práctica" ( Schön,1998: 124). El mejor modo para resolver estos problemas especícos, propone el autor, es "atendiendo a la reexión desde la acción de los profesionales"(Schön, 1998: 124), allí, es posible descubrir una estructura fundamental de la indagación profesional, en donde se deja sentir una heurística, acompañada de creatividad, acción pedagógica y artística con matices complejos.

Dentro de los elementos integrales de Schön, que comprenden la estructura del ejercicio reexivo desde una acción pedagógica y que podrían aportar a la generación de ciencia estarían:

"<la presentación de una situación única e incierta>; <entablar conversación>; <intento del estudiante por resolver el problema>; <participación del profesor, el cual saca a ote y critica lo encuadrado por el estudiante>; a su vez y de forma implícita, $<$ el docente deja que su crítica del viejo problema sea inferida a partir de su modo de restructurar >; <el estudiante entra en deducción integralmente con su paciente>; $<$ el profesor reformula el problema y sugiere una dirección para reorganizar la situación>; <el estudiante es incitado a imponer una geometría> <el profesor pide que el estudiante se ponga sobre la realidad obtenida, que haga parte de la misma>; <el profesor toma el problema reestructurado y dirige un experimento tendente a descubrir qué consecuencias e implicaciones pue- 
den hacerse a partir de él>(...)" (Schön, 1998: 125-126).

Es de notar que la práctica pedagógica implícitamente encierra una complejidad, una estructura íntimamente relacionada y un rigor que conlleva a hallar la luz, lo nuevo, el cambio que conlleva al agradecimiento y por consiguiente al ser feliz. "(...) Finalmente las acciones producen cambios intencionados que proporcionan nuevos signicados que requieren una nueva reexión desde la acción o práctica"(Schön,1998: 126).

"Es un proceso en espiral a través de etapas de apreciación, acción, y reapreciación" ( Schön,1998: 126), dentro de este proceso, la situación llega a ser comprendida a través del deseo y acción de cambio; es de esta forma que se genera la epistemología en la práctica. Desde aquí el profesional construye gradualmente ciencia, a partir de su percepción, exposición, yendo hacia una práctica e interpretación congruente de valores y teorías fundamentales.

Dentro de este contexto epistemológico de reexión acción, el experimento es muy necesario; "la experimentación es una actividad mediante la cual un investigador conrma o refuta una hipótesis"(Schön,1998: 133). Esta idea permite ver que el experimento se equipara al producido por la racionalidad técnica, en cuanto que busca apoyarse en la teoría cientíca y ésta, a su vez confrontarse y reproducirse mediante la pedagogía. "Hoy nos gusta separar todo(...) creemos que no hay ninguna relación entre la geometría y la política(...)" ( Ospina, 2012:29). Se olvidó que ciencia y pedagogía son complementarias. De modo que hay ciencia en cuanto la pedagogía promueve una reexión ecaz que conlleva estratégicamente a un ejercicio o práctica contundente que promueve cambios subjetivos y objetivos.

Experimentar es actuar operativamente para ver a qué conduce la acción dentro de las prácticas en aras a un resultado. Sin embargo, se ha de evitar al máximo caer en acciones que solamente buscan ver qué sigue, sin el acompañamiento de predicciones, que han de emanar de la actividad pedagógica y que de no ser así, se constituiría en un experimento exploratorio, en un procedimiento formal que las ciencias positivas promueven. "El experimento exploratorio es el sondeo, la actividad juguetona mediante la cual tenemos cierta sensación respecto a las cosas. Tiene éxito cuando conduce al descubrimiento de alguna cosa que está ahî" ( Schön,1998: 137).

Jugar inicialmente con la práctica, ha de ser nalmente la actividad propia de cada docente con sus estudiantes, para decantar un conocimiento con estrategias didácticas que favorezcan el ir y venir de un conocimiento que poco a poco se acrisola con la práctica donde la reexión es imprescindible, de no ser así, se estaría sobre un cumplimiento formal que enmarca un currículum en el que el deseo de novedad es muy lejano y la prevalencia del concepto se agudiza, por consiguiente imposible hacer ciencia.

Se ha de considerar, por consiguiente, que el camino cientíco lo deben emprender los mismos pedagogos o educadores ya que son ellos quienes cuentan con un sinnúmero de posibilidades a desarrollar con sus estudiantes. Así " $\mathrm{La}$ educación se puede entender como trasmisión de conocimientos, también podríamos entenderla como búsqueda y transformación del mundo en que vivimos" ( Ospina, 2012: 30). Por esto mismo, se ha de recuperar el espacio investigativo en la escuela, en el colegio e imprescindible en la universidad, de modo que se constituyan verdaderamente en lugares de estudio, de investigación y de producción pedagógica y de ciencia, porque "somos cántaros llenos que habría que vaciar un poco, para que vayamos remplazando tantas vanas certezas por algunas preguntas provechosas." (Ospina, 2012:25) preguntas que por ningún motivo han de comunicar respuestas denitivas. En últimas, es de considera que la pedagogía es el camino por el que viaja la ciencia, camino que se identica con un ám- 
bito de partida más su destino es indeterminado.

\section{CONCLUSIÓN}

La dinámica racional, promovida por la modernidad dicultó, complejizó, por no decir que extravió la misión y dinámica de la educación. En consecuencia con Kant (revolución copernicana), en la modernidad ya no es el "Ser" quien impacta, conmueve o promueve sentimientos en la especie humana, sino que por el contrario, es la especie humana quien impacta, conmueve, transforma e imprime efectos en el "Ser". Desde esta perspectiva, con la modernidad hasta hoy, el ser humano ha devenido en objeto $\mathrm{y} n$ de sus actos al mismo ser humano. De ahí, resulta válido considerar que la educación se alía a la razón a $n$ de resolver problemas más de carácter cientíco técnico, que humanos.

Por lo anterior, este artículo exhorta al retorno del ejercicio de la educación en tanto misión natural, esto es, educar a la especie humana, la cual es imposible educar sin el uso apropiado de la pedagogía, por una razón muy sencilla, mientras la educación sin pedagogía adiestra para resolver problemas de producción y consumo, formando sujetos pasivos; la educación con pedagogía, enseña y promueve para la vida y forma sujetos activos, motivo por el cual se aprenden a resolver problemas y a generar conocimiento. Esto último es la razón del porqué implicar la pedagogía en las prácticas educativas; es de una u otra forma aproximarnos a hacer ciencia, en cuanto que problemas y reexión emergen a su paso creando o recreando motivos de vida para nuevos días.

\section{BIBLIOGRAFÍA}

Bourdieu, P. y Passeron J.C. (1981). La Reproducción. Barcelona: Laia.

Bourdieu, P. (2008). Homo Academicus. Argentina, XXI.

Dewey, J. (2004). Democracia y educación. Madrid: Morata.

Holmes Beck Robert. (1965). Historia Social de la Educación. (Traducido al español por Gerhard Carlos) México: Utea.

Kuhn, T.S. (2012) Historia de la losofía. En: http://www. webdianoia.com/contemporanea/kuhn.htm Rescatado en octubre 02 de 2012.

http://w ww.ban taba.ehu.es/f or marse/fiche ros/view/Modelos_Educativos.pdf?revision_ $i d=80120 \&$ package_id=80004/ (13 de julio 2012.)

http://www.eltiempo.com/opinion/editoriales/ARTICU LO-WEB-NEW_NOTA_INTERIOR-12135934.html (7:38 p.m./16 de Agosto del 2012)

Ospina, W. (2012). La Lámpara Maravillosa. Cuatro ensayos sobre la educación y un elogio de la lectura. Colombia: Mondadori.

Popper, K.R., (1977) La lógica de la investigación cientíca. Madrid: Tecnos.

Schön D. (1998). El Profesional Reexivo. Cómo piensan los profesionales cuando actúan. Barcelona: Paidós.

Unigarro, G. Manuel A. (1999). Introducción a las Teorías Educativas Contemporáneas. Bucaramanga- Colombia, Unab.

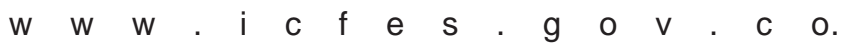

Principales resultados en los módulos de competencias genéricas Rescatado el 17 de Abril de 2012).

Cómo citar este artículo:

Bautista, M. E. (2012) La pedagogía en el umbral de la ciencia. Espiral, Revista de Docencia e Investigación. $2,(1), 65-78$ 
